\title{
Crossing integer spin resonance with conservation of beam polarization
}

\author{
A. K. Barladyan, ${ }^{1}$ A. Yu. Barnyakov, ${ }^{1,2}$ V. E. Blinov, ${ }^{1,2,3}$ S. A. Glukhov, ${ }^{1}$ S. E. Karnaev, ${ }^{1}$ \\ E. B. Levichev, ${ }^{1,3}$ S. A. Nikitin, ${ }^{1, *}$ I. B. Nikolaev ${ }^{1,2, \dagger}$ I. N. Okunev, ${ }^{1}$ \\ P. A. Piminov, ${ }^{1}$ A. G. Shamov, ${ }^{1,2}$ and A. N. Zhuravlev ${ }^{1,2}$ \\ ${ }^{1}$ Budker Institute of Nuclear Physics, 11, Lavrentiev prospect, Novosibirsk, 630090, Russia \\ ${ }^{2}$ Novosibirsk State University, 2, Pirogova street, Novosibirsk, 630090, Russia \\ ${ }^{3}$ Novosibirsk State Technical University, 20, Karl Marx prospect, Novosibirsk, 630092, Russia
}

(Received 3 July 2019; published 20 November 2019)

\begin{abstract}
A method proposed to preserve the electron beam polarization at the VEPP-4M collider during acceleration with crossing the integer (imperfection) spin resonance at energy $E=1763 \mathrm{MeV}$ has been successfully applied. It is based on full decompensation of the $0.6 \times 3.3$ Tesla $\times$ meter integral of the KEDR detector longitudinal magnetic field due to the antisolenoid being "switched off."
\end{abstract}

DOI: $10.1103 /$ PhysRevAccelBeams.22.112804

\section{MOTIVATION}

The set of the beam energy values in the hadron-muon branching ratio measurement with the KEDR detector [1] at the electron-positron VEPP-M collider [2] in the region between $J / \psi$ and $\psi^{\prime}$ resonances includes several critical points, in particular, $E=1764$ and $1814 \mathrm{MeV}$. The beam energy calibration in this experiment is performed using the resonant depolarization technique (RD), and thus polarized beams are required. Polarization is obtained due to the natural radiation mechanism at the VEPP-3 booster storage ring (see Fig. 1). Both of these energy values are in the so-called "polarization downfall" (Fig. 2), which is the VEPP-3 energy range with a width of approximately $160 \mathrm{MeV}$. In this range, obtaining polarization is significantly difficult due to the strong depolarization effect caused by various field imperfections. The polarization downfall range was found in the 2003 year experiment with the polarimeter based on an internal polarized target [3].

The center of that critical range is the energy value $E_{4}=1763 \mathrm{MeV}$, which corresponds to the fourth imperfection spin resonance $\nu=\nu_{k}=4$. In a conventional storage ring without any imperfections, $\nu=\gamma a$ is the spin tune parameter equal to the number of the spin vector precessions about the vertical guide field per a turn minus one; $\gamma$ is the Lorentz factor; $a=(g-2) / 2$ is the magnetic dipole moment anomaly. Nevertheless, one can obtain

\footnotetext{
*S.A.Nikitin@inp.nsk.su

†.B.Nikolaev@inp.nsk.su
}

Published by the American Physical Society under the terms of the Creative Commons Attribution 4.0 International license. Further distribution of this work must maintain attribution to the author(s) and the published article's title, journal citation, and DOI. polarized beams at VEPP-4M with the energies from the polarization downfall, except for a small island in the vicinity of $E_{4}$ using the method of "advance energy point." In this experiment, the magnetization reversal cycle of the collider is of the "upper" type. This means that the advance energy points should be below the energies of experiment, as well as below $1660 \mathrm{MeV}$, i.e., the lower boundary of the polarization downfall region. In the cases when the method is valid, the beam polarization in VEPP-3 is achieved at the "advance" energy. Then the beam is injected into the collider ring. After that its energy is raised to the energy of the experiment. The radiative spin relaxation time in the collider ring is 2 orders larger than that in the booster $\operatorname{ring}\left(\tau_{p} \approx 80 \mathrm{~h}\right.$ at $\left.E=1.8 \mathrm{GeV}\right)$. This allows us to apply the RD technique even at rather small detuning from the dangerous spin resonances, despite the sharp increase in the depolarizing effect of quantum fluctuations in the presence of guide field imperfections. For instance, it was a case in the tau-lepton mass measurement [4] at energies close to the tau production threshold $(E=1777 \mathrm{MeV})$. The RD calibrations were carried out at the detuning $\delta \nu \approx 0.03$ ( $\Delta E \approx 13 \mathrm{MeV}$ in units of energy) and less from the resonance $\nu_{k}=4$. The magnetization reversal cycle of the "lower" type was used, and the advance energy was $1.85 \mathrm{GeV}$. After the beam was injected into VEPP-4M, its energy decreased to the energy of the tau production threshold.

In the case under consideration, one could apply a similar method at energy points below $1763 \mathrm{MeV}$. However, there was a need for special measures in relation to the energy $E=1814 \mathrm{MeV}$, as well as to the points of energy somewhat below the $\psi^{\prime}$ peak (for example, $E=1839 \mathrm{MeV}$ ). The reason was the necessity to cross the integer spin resonance at $1763 \mathrm{MeV}$ during acceleration starting from the advance energy. 


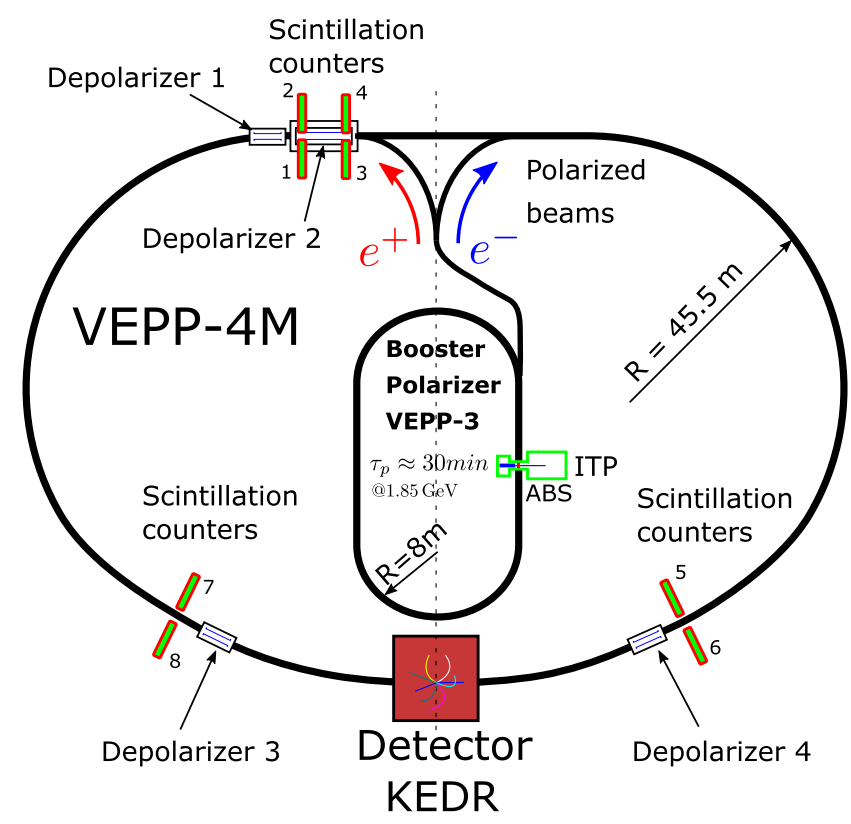

FIG. 1. Scheme of the VEPP-4 complex from the point of view of polarization experiments. ITP is the internal target setup based on the use of a jet of polarized deuterium atoms from the Atomic Beam Source (ABS).

\section{ISSUES OF FAST/SLOW CROSSING}

A sufficiently high rate of beam acceleration or deceleration in a storage ring can save the polarization of particles to a considerable extent in the crossing of any spin resonance $\nu_{0}=\nu_{k}$. Generally, an actual spin tune $\nu_{0}$ differs from the parameter $\nu$ defined for storage rings with a unidirectional guide field. For fast crossing, the following condition must be fulfilled $[5,6]$ :

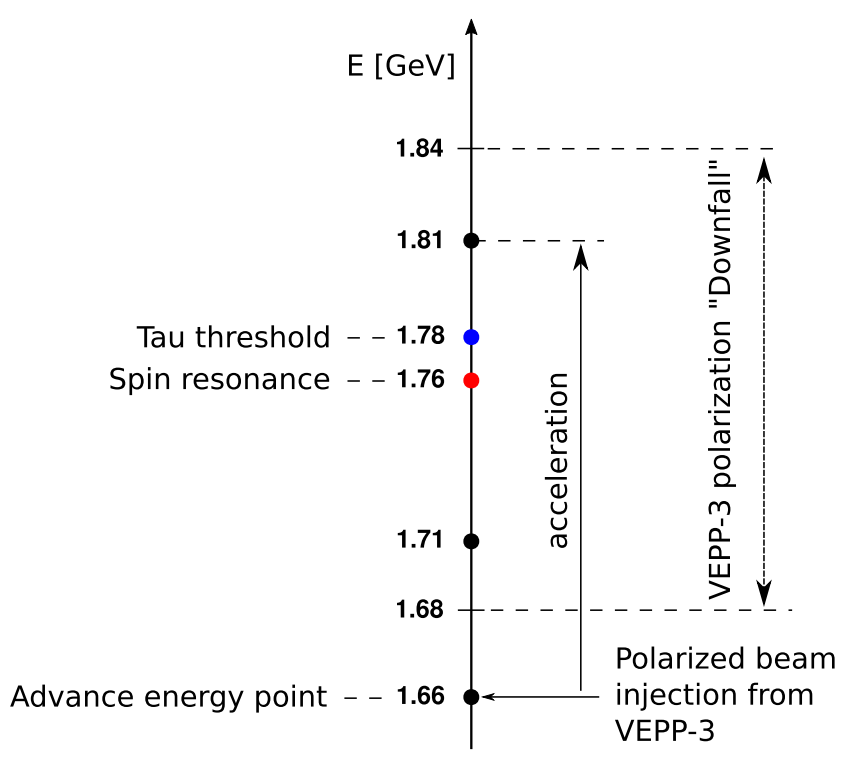

FIG. 2. Diagram of energy points in the experiment.

$$
\frac{d \varepsilon}{d t}=\dot{\varepsilon} \gg\left|w_{k}\right|^{2} \omega_{0}
$$

where $\varepsilon(t)=\left|\nu_{0}(t)-\nu_{k}\right|$ is the time-dependent resonant detuning; $w_{k}$ is the resonant harmonic amplitude of the field perturbations; $\omega_{0}=2 \pi f_{0}$ is the angular frequency of particle revolution (at VEPP-4M, $f_{0}=819 \mathrm{kHz}$ ). The corresponding loss in the degree of polarization at a single crossing of the resonance is $\sqrt{\pi\left|w_{k}\right|^{2} \omega_{0} / \dot{\varepsilon}} \ll 1$. The data on the polarization lifetime $\tau_{d}$ due to radiative depolarization in the vicinity of $E=1777 \mathrm{MeV}$, obtained during the preparation of the tau-lepton mass measurement, contain the information about the natural strength of the spin resonance $\nu_{k}=\nu=4$. The polarization lifetime was adjusted to the level $\tau_{d} \geq 1 \mathrm{~h} \mathrm{[7].} \mathrm{One} \mathrm{can} \mathrm{associate} \mathrm{this}$ quantity with the formal estimate of the resonant spin harmonic amplitude using the known equation [8,9]:

$$
\tau_{d} \approx \frac{\tau_{p}}{1+\frac{11}{18} \frac{\left|w_{k}\right|^{2} \nu^{2}}{\varepsilon^{4}}},
$$

where $\varepsilon=\nu_{0}-4 \approx 0.03 \ll 1$, and $\tau_{p}$ is the SokolovTernov polarization time [10]. For VEPP-4M, at $E=$ $1777 \mathrm{MeV}, \tau_{p}=87 \mathrm{~h}, \tau_{d}=1 \mathrm{~h}$, and $\varepsilon \approx 0.03$; so the estimate is $\left|w_{k}\right| \sim 2.8 \times 10^{-3}$. Therefore, the maximum necessary rate of the resonance crossing is $\dot{\varepsilon} \gg 20 \mathrm{~s}^{-1}$, or $d E / d t \gg 10^{4} \mathrm{MeV} / \mathrm{s}$. In practice, the achievable ramping rate at VEPP-4M does not exceed 10-20 MeV/s. So, fast crossing of the integer spin resonance $E=1763 \mathrm{MeV}$ is impossible.

If the ramping rate is reduced so that the following condition $[5,6,8]$

$$
\dot{\varepsilon} \ll\left|w_{k}\right|^{2} \omega_{0}
$$

is satisfied, then the spin resonance intersection occurs adiabatically slowly. Basing on the estimates made above, one can conclude that, in principle, a rate of $1-10 \mathrm{MeV} / \mathrm{s}$ may be appropriate. In the theoretical limit of the adiabatic crossing without taking into account the radiation effects, the polarization retains its value and changes the sign (the spin flip mode).

Despite the feasibility of the condition of slow crossing, it is necessary to bear in mind that there is a lower limit on the rate of the crossing because of the depolarizing effect of radiation diffusion and damping. The radiation depolarization time related to the vertical closed orbit distortions declines very quickly with the detuning from the integer spin resonance: $\tau_{d} \propto \varepsilon^{4}$. For example, this time decreases by a factor of 16 at $E=1770 \mathrm{MeV}$ as compared with $\tau_{d}=1 \mathrm{~h}$ at $E=1777 \mathrm{MeV}$. In the case of strong resonance, the spin diffusion also depends on the decrement $\Lambda$, the parameter of radiation damping. While passing the resonant region $|\varepsilon| \sim\left|w_{k}\right|$ at $\omega_{0}\left|w_{k}\right| \gg \Lambda$, due to radiation diffusion and damping, the depolarization time reaches the 
minimum value of $\tau_{d} \sim \Lambda^{-1} \sim 100 \mathrm{~ms}$ [11]. The estimates given in [12] allow us to conclude that the adiabatic crossing of the forth spin resonance in VEPP-4M will lead to a notable loss of beam polarization.

\section{SPIN KINEMATICS AT KEDR DETECTOR FIELD DECOMPENSATION}

A simple approach to preserve the VEPP-4M beam polarization in the conditions under consideration was proposed and numerically substantiated in [12] and then successfully implemented in the experiment [13]. It is based on the well-known idea of a partial Siberian snake [14].

If one switches off the current in the antisolenoid coils, the 0.6 Tesla longitudinal magnetic field integral of the KEDR detector becomes uncompensated, which results in the rotation of the spin around the velocity by an angle $\varphi$. In particular, $\varphi \approx 0.34 \mathrm{rad}$ at $E=1.75 \mathrm{GeV}$.

Figure 3 illustrates the principle difference between the cases of a storage ring without and with an arbitrary solenoid of $\varphi \neq 0$ at the condition $\nu=$ integer. We assume that initially there are no field perturbations in the hypothetical storage ring. In the first case (a), through a revolution of a particle, any direction of the polarization vector turns into itself. By this reason, the spin perturbations growing due to fluctuations and the spin tune spread lead to fast breaking of the beam polarization. In the second case (b), there exists a general equilibrium periodical direction of polarization, the $\vec{n}$ vector, for all beam particles. This vector rotates in the median plane and is directed along the particle velocity at the azimuth where the solenoid is located. Spins with deviations from $\vec{n}$ make precession around it. Owing to this fact, the spin motion is stabilized largely. Depolarization can occur through relatively slow accumulation of small changes in the average projection of the spins on the axis $\vec{n}$.

A longitudinal magnetic field causes a shift of the spin tune with regard to an unperturbed value $\nu$. Thus, the noninteger part of the perturbed spin frequency $\nu_{0}$ does not take a zero value at the critical point near $E=1763 \mathrm{MeV}$. (a)

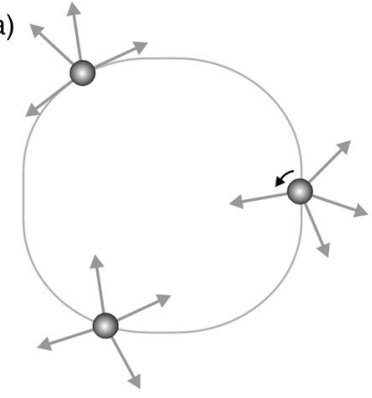

(b)

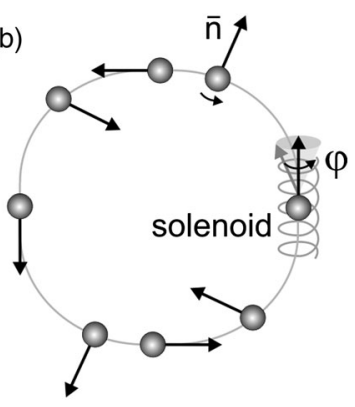

FIG. 3. Two cases of the spin kinematics at $\nu=$ integer. (a) There is no preferable direction of the spin polarization in an ideal storage ring with a flat orbit. (b) There is a dynamically stable vector $\vec{n}$, the periodical precession axis, when an arbitrary solenoid is introduced.
This fact is used as a basis for preserving the beam polarization during acceleration.

The equilibrium polarization axis as a function of the azimuth $\vartheta$ in a storage ring having at $\vartheta=0$ an insertion with longitudinal magnetic field is calculated using the known formulas $[15,16]$ :

$$
\begin{aligned}
n_{x}(\vartheta) & = \pm \frac{\sin \nu(\vartheta-\pi)}{\sin \xi} \cdot \sin \frac{\varphi}{2} \\
n_{y}(\vartheta) & =\mp \frac{\cos \nu(\vartheta-\pi)}{\sin \xi} \cdot \sin \frac{\varphi}{2} \\
n_{z}(\vartheta) & =\mp \frac{\sin \pi \nu}{\sin \xi} \cdot \cos \frac{\varphi}{2} \\
\sin \xi & =\sqrt{1-\cos ^{2} \pi \nu \cos ^{2} \frac{\varphi}{2}}
\end{aligned}
$$

Here

$$
\varphi \approx \frac{\pi}{4.6 \nu} \cdot \int H_{\|} d s
$$

is the angle of electron spin rotation in the longitudinal magnetic field with the integral of $\int H_{\|} d s$ in Tesla $\cdot$ meter. The symbols $x, y$, and $z$ mark the horizontal, longitudinal, and vertical axes of the Frenet-Serret coordinate system, respectively. We use an approximation of an isomagnetic storage ring in which the azimuth $\vartheta$ equals the angle of the particle velocity rotation. The effective spin precession tune is determined from the equation

$$
\cos \pi \nu_{0}=\cos \pi \nu \cos \frac{\varphi}{2} .
$$

In our case, $\int H_{\|} d s=H_{\mathrm{KEDR}} \cdot L_{\mathrm{eff}}$, where $H_{\mathrm{KEDR}}=$ 0.6 Tesla, the KEDR detector field; the effective KEDR solenoid length $L_{\text {eff }}=3.3 \mathrm{~m}$ if the antisolenoids are switched off. Figure 4 shows the calculated spin tune shift

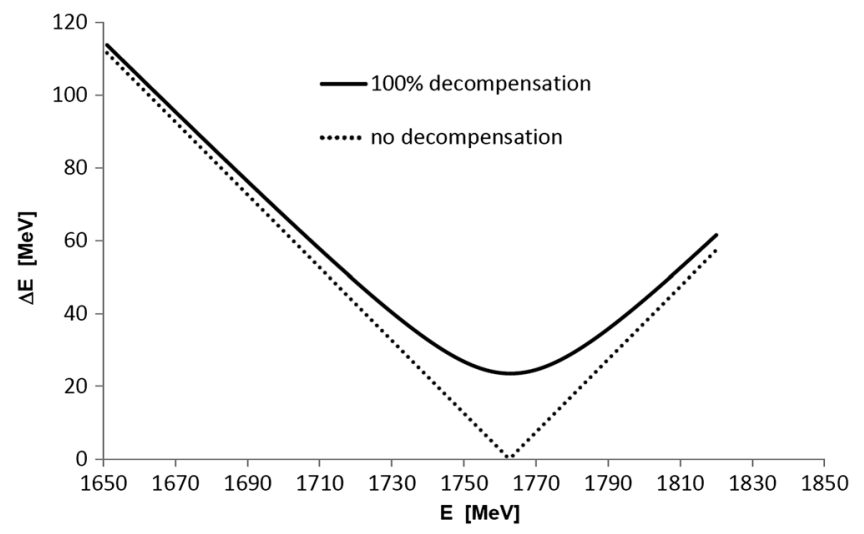

FIG. 4. Spin tune shift in the energy units vs the beam energy in the cases of no and full decompensation of the KEDR detector field integral of $0.6 \times 3.3$ Tesla $\times$ meter. 
$\left|\nu_{0}-\nu\right| \propto \Delta E$ versus the beam energy at full decompensation. This shift is about $\Delta E=22 \mathrm{MeV}$ in the vicinity of the critical energy $1763 \mathrm{MeV}$.

\section{COMBINATION SPIN RESONANCES}

In our experiment, there is an intersection of some combination spin resonances $\nu_{0} \approx \nu=\nu_{k}$, which depend on the betatron tunes $\nu_{x}=8.536$ and $\nu_{z}=7.572$, as well as the synchrotron tune $\nu_{\gamma} \approx 0.01$. Because of the relatively narrow range of energy adjustment, the main spin-betatron resonances $\nu \pm \nu_{z}=k_{z}$ and $\nu \pm \nu_{x}=k_{x}$ did not fall into it. According to the results of the experiment, it can be argued that the weaker spin-betatron resonances $\nu+\nu_{x}+\nu_{z}=20$ (1715 MeV), $\nu-\nu_{x}-\nu_{z}=-12(1810.2 \mathrm{MeV})$, and the resonances due to synchrotron modulation $\nu \pm 5 \nu_{\gamma}=4$ (1740.6 and $1784 \mathrm{MeV}$ ) were successfully crossed. To comment on this, below are estimates of the strength of these resonances.

We estimate the amplitude of the harmonic of the spin resonances of the $\nu \pm \nu_{x} \pm \nu_{z}=k_{x z}$ type from the formula

$$
\left|w_{k_{x z}}\right| \sim\left|\left\langle\nu h \sigma_{x} \sigma_{z} F^{\nu} e^{i\left[ \pm\left(\mu_{z}+\mu_{x}\right) \mp\left(\nu_{x}+\nu_{z} \pm k_{x z}\right) 9\right]}\right\rangle\right| \sim 10^{-7} .
$$

Here, $h=\partial^{2} H_{z} / \partial x^{2}$ is the quadratic nonlinearity due to the sextupole correction (in the units of the mean field $\left.\left\langle H_{z}\right\rangle\right) ; \sigma_{x}$ and $\sigma_{z}$ are the transverse beam sizes; $\mu_{x, z}$ are the betatron phase advances; $\langle\cdots\rangle$ is averaging over azimuth; $F^{\nu}(\vartheta)$ is the periodic spin response function [8]. The function $F^{\nu}$ takes into account the depolarization effect of vertical betatron oscillations excited by any local perturbation. The results of the calculation of this characteristic for VEPP-4M are given, for example, in [17]. Fast crossing of such weak resonances becomes possible, starting from the very low rates of the energy change: $d E / d t \gg 100 \mathrm{eV} / \mathrm{s}$.

The strength of the $m$ th-order modulation resonance related to synchrotron oscillations depends on the amplitude $\Delta$ of spin tune modulation. The corresponding amplitude of the resonant harmonic of any spin perturbations $w_{k m}$ is associated with the fundamental harmonic $w_{k}=w_{k 0}$ through the equation

$$
\left|w_{k m}\right|=\left|w_{k}\right| \cdot J_{m}\left(\frac{\Delta}{\nu_{\gamma}}\right),
$$

$J_{m}$ being the Bessel function. For estimation, let $\Delta=$ $\sqrt{2}\left|\gamma \partial \nu_{0} / \partial \gamma\right| \sigma_{\gamma}$, where $\gamma \partial \nu_{0} / \partial \gamma$ is the spin tune chromaticity; $\sigma_{\gamma}$ is the beam energy spread $\left(\approx 3 \times 10^{-4}\right.$ at $\left.1.8 \mathrm{GeV}\right)$. In the case of a single solenoid, which dominates over other imperfections, the chromaticity is found as

$$
\gamma \frac{\partial \nu_{0}}{\partial \gamma}=\frac{\nu}{\sin \pi \nu_{0}}\left(\sin \pi \nu \cos \frac{\varphi}{2}-\frac{\varphi}{2 \pi \nu} \cos \pi \nu \sin \frac{\varphi}{2}\right) .
$$

For example, at $\varphi=0$, the spin tune chromaticity is just $\nu$ (the conventional storage ring with $\nu_{0}=\nu$ ). In the Siberian snake scheme ( $\left.\varphi=\pi, \nu_{0}=1 / 2\right)$, this parameter is equal to $(-1 / 2) \cos \pi \nu$. At $\varphi \ll 1$ and $\nu=k$,

$$
\gamma \frac{\partial \nu_{0}}{\partial \gamma} \approx-\frac{\varphi^{2}}{4 \pi \sin \pi \nu_{0}} \approx-\frac{\varphi^{2}}{4 \pi^{2} \varepsilon},
$$

where $\varepsilon=\nu_{0}-k$. In our case, $\varphi=0.34, \varepsilon \approx 0.1(k=4)$, $m=5, \Delta \approx 10^{-5},\left|w_{k 5}\right| /\left|w_{k}\right| \sim 10^{-17}$. The crossing of this sideband resonance means nothing for polarization.

\section{RADIATIVE DEPOLARIZATION RATE DURING ACCELERATION}

The characteristic time $\tau_{d}$ of the radiative depolarization due to quantum fluctuations in the presence of a strong perturbation in the form of the KEDR detector longitudinal field can be found from the generalized equation [9]:

$$
\tau_{d} \approx \frac{\tau_{p}}{\left\langle 1-\frac{2}{9}(\vec{n} \vec{\beta})^{2}+\frac{11}{18} \vec{d}^{2}\right\rangle} .
$$

Here $\tau_{p}$ is the Sokolov-Ternov polarization time (it is proportional to $E^{-5}$ and amounts to $72 \mathrm{~h}$ at the VEPP-4M energy of $1.85 \mathrm{GeV}$ ); $\vec{d}^{2}$ is the square of the periodical spinorbit coupling vector function of the azimuth; $\vec{\beta}$ is the particle velocity relative to the speed of light. In our case, the spin-orbit coupling is excited by the uncompensated part of the KEDR field integral. It can be found as the derivative of vector (4) with respect to the Lorentz-factor $\gamma$ of particles [16]:

$$
\begin{aligned}
d_{x}= & \gamma \frac{\partial n_{x}}{\partial \gamma}= \pm\left\{F \sin \nu(\vartheta-\pi) \cdot \sin \frac{\varphi}{2}\right. \\
& +\frac{1}{\sin \xi}\left[\nu(\vartheta-\pi) \cdot \cos \nu(\vartheta-\pi) \cdot \sin \frac{\varphi}{2}\right. \\
& \left.\left.-\frac{\varphi}{2} \cdot \sin \nu(\vartheta-\pi) \cdot \cos \frac{\varphi}{2}\right]\right\}, \\
d_{y}= & \gamma \frac{\partial n_{y}}{\partial \gamma}=\mp\left\{F \cos \nu(\vartheta-\pi) \cdot \sin \frac{\varphi}{2}\right. \\
& -\frac{1}{\sin \xi}\left[\nu(\vartheta-\pi) \cdot \sin \nu(\vartheta-\pi) \cdot \sin \frac{\varphi}{2}\right. \\
& \left.\left.+\frac{\varphi}{2} \cdot \cos \nu(\vartheta-\pi) \cdot \cos \frac{\varphi}{2}\right]\right\}, \\
d_{z}= & \gamma \frac{\partial n_{z}}{\partial \gamma}=\mp\left\{F \sin \pi \nu \cdot \cos \frac{\varphi}{2}\right. \\
& \left.+\frac{1}{\sin \xi}\left[\pi \nu \cdot \cos \pi \nu \cdot \cos \frac{\varphi}{2}+\frac{\varphi}{2} \cdot \sin \pi \nu \cdot \sin ^{2} \frac{\varphi}{2}\right]\right\}, \\
F= & -\frac{1}{2 \sin ^{3} \xi}\left(\pi \nu \sin 2 \pi \nu \cdot \cos ^{2} \frac{\varphi}{2}-\frac{\varphi}{2} \sin \varphi \cdot \cos ^{2} \pi \nu\right) .
\end{aligned}
$$


Formula (5) is valid outside of spin resonances. We use it together with the set of equations (6) to calculate the radiative diffusion of polarization during acceleration, since in the presence of a solenoid, the effective spin precession frequency does not take integer values.

Another contribution to the spin-orbit coupling is given by the betatron oscillations excited by quantum fluctuations. In consideration of various cases with spin rotators based on solenoids, the contribution of betatron oscillations far from the spin-betatron resonances to the depolarization rate is small as compared with the effect of the dependence of the polarization axis $\vec{n}$ on the energy [16,18-20]. For this reason we neglect the betatron oscillations.

The depolarization time is calculated using formulas (5) and (6) and plotted in Fig. 5 versus the beam energy at $100 \%$ and $50 \%$ decompensation of the KEDR field integral. The minimum depolarization time $\tau_{d}=20 \mathrm{~s}$. The width of the energy area where $20 \mathrm{~s}<\tau_{d}<100 \mathrm{~s}$ is about $30 \mathrm{MeV}$. It takes about $30 \mathrm{~s}$ to cross this area at a nominal rate of energy change $d E / d t=1 \mathrm{MeV} / \mathrm{s}$.

At $1810 \mathrm{MeV}$, the time $\tau_{d}$ becomes long enough, $2000 \mathrm{~s}$. This gives a chance to measure the energy from the spin frequency using the RD technique after the end of the acceleration process, followed by the restoration of the field in the antisolenoids.

The theoretical behavior of the polarization degree during acceleration from the injection (advance) energy $E=1650 \mathrm{MeV}$ is shown in Fig. 6 for two values of the acceleration rate. The current value of the degree in the units of the initial one $\left(P_{0}\right)$ is calculated from the equation

$$
\frac{P}{P_{0}} \approx \exp \left[-\int_{E_{1}}^{E_{2}} \frac{d E}{(d E / d t) \cdot \tau_{d}}\right]
$$

It is seen that it is advantageous to apply the full decompensation of the KEDR field and perform acceleration with a rate not below $2 \mathrm{MeV} / \mathrm{s}$. In the best case, it can

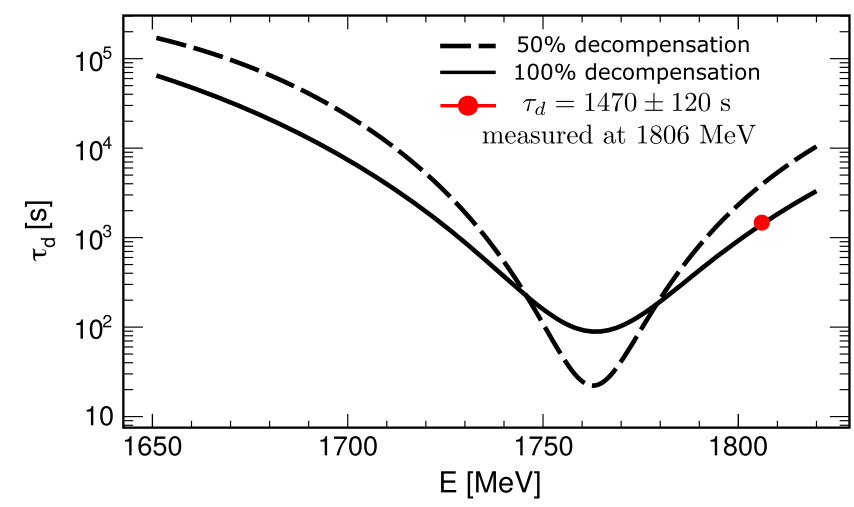

FIG. 5. The calculated radiative depolarization time vs the beam energy under the influence of 0.6 T KEDR field decompensation. The separate point shows the measured value at an energy of $1806 \mathrm{MeV}$ (estimated value of $1407 \mathrm{~s}$ ).

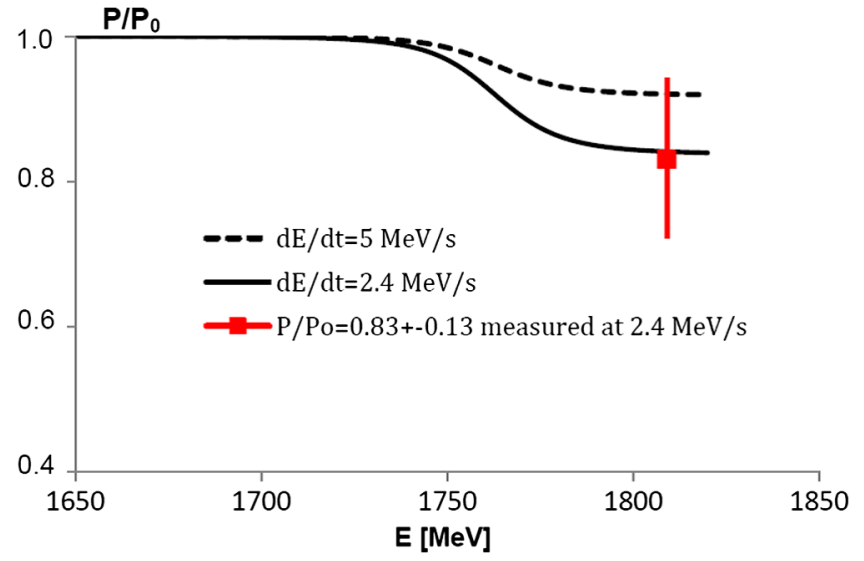

FIG. 6. The calculated change in the degree of polarization relative to the initial one in the course of beam acceleration at a rate characterized by the parameter $d E / d t$ in the case of complete decompensation of the KEDR field. The separate point with the error bars presents the generalized experimental data.

provide about $80 \%$ of the initial polarization degree in the final state. RD calibration of the beam energy should be performed only after restoration of the antisolenoid field, which leads to the elimination of the spin tuning shift, and with it a systematic error in the energy value.. Moreover, the polarization lifetime increases manifold if the KEDR field is compensated.

\section{COMPENSATION OF BETATRON COUPLING FROM KEDR FIELD}

If the antisolenoids are switched off, the special measures are needed to provide the relevant alternative operation modes of VEPP-4M. It is convenient to use the scheme of betatron coupling localization by Klaus Steffen [21], which includes two skew quadrupole lenses (Fig. 7).

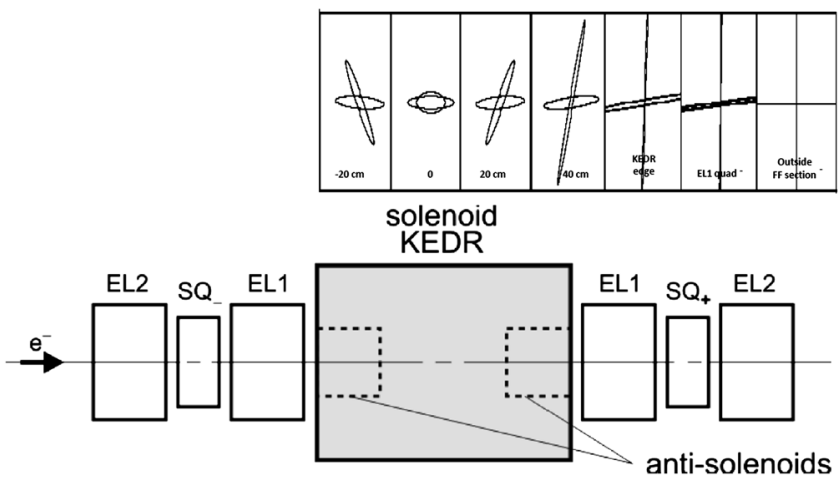

FIG. 7. The scheme (depicted not to scale) of compensation of the betatron coupling caused by the KEDR main solenoid field with the help of two skew quadrupoles (SQ+ and SQ-) located near the final focus lenses. The antisolenoids are off. Above is a view of normal betatron modes in the $\mathrm{X}-\mathrm{Z}$ plane at different distances from the center of the detector. 
This scheme has already been successfully tested at VEPP-4M [22,23].

The transport matrix for the vector of betatron variables $\left(x, x^{\prime}, z, z^{\prime}\right)$ in the section from the skew lens $\mathrm{SQ}_{+}$to $\mathrm{SQ}_{-}$ including KEDR can be approximately written as

$$
M=Q_{-} \cdot M_{-} \cdot M_{s} \cdot L^{2} \cdot M_{s} \cdot M_{+} \cdot Q_{+} .
$$

Here $Q_{ \pm}$are the matrices of "thin" skew squads; $L$ is the drift section matrix of length $l=L_{s} / 4 ; L_{s}=3.3 \mathrm{~m}$ is the effective length of the KEDR main solenoid $\left(L_{s}=2.5 \mathrm{~m}\right.$ when the antisolenoids are switched on); $M_{s}$ is the halfsolenoid matrix in the "thin magnet" approximation $(\chi=\varphi / 2)$ :

$$
M_{s}=\left(\begin{array}{cccc}
1 & 0 & -\chi & 0 \\
0 & 1 & 0 & -\chi \\
\chi & 0 & 1 & 0 \\
0 & \chi & 0 & 1
\end{array}\right)
$$

$M_{ \pm}$are the matrices for transformation from the center of the right (left) half of the KEDR solenoid to the corresponding skew quad. The skew quads are placed symmetrically relative to the solenoid in the "magic" azimuths, for which some elements of the matrix $M$ exactly or approximately satisfy a certain equation. The focusing strengths of the lenses $S Q_{ \pm}$are found from another equation. They are proportional to $\chi$, close in value and opposite in sign. With these lenses in mind, the $M$ matrix will not contain off-diagonal blocks $2 \times 2$ or will be close to this form. The simplicity of the scheme is based on the mirror symmetry of the magnetic structure in the section with the solenoid. The betatron coupling is localized in this section. The vertical and horizontal oscillations excited beyond the section are mutually independent within the accuracy of the compensation scheme design and realization. The scheme provides a minimum split of the normal betatron mode frequencies of the order of $10^{-3}$ (in the units of the revolution frequency). If no compensation is applied, this split achieves 0.1 , and this hampers sustainable maintenance of the beam during acceleration.

\section{TOUSCHEK POLARIMETER}

To observe the beam polarization, a system of absolute calibration of the beam energy by the frequency of the spin precession was used [24,25]. The system includes the Touschek polarimeter, based on the intrabeam-scattering (IBS) effect, and the TEM wave-based depolarizer. The polarimeter consists of eight plastic scintillator counters, pushed inside the accelerator vacuum chamber to registrate the particles scattered from the beam (Fig. 1). The rate of counting IBS events depends on the square of beam polarization [26-29]. In the depolarizer, the TEM wave is generated with the help of two parallel, vertically spaced conductive plates connected to a variable-frequency $\mathrm{rf}$ generator. We have several such devices located at different azimuths of the VEPP-4M ring. In various experiments, the most suitable device is used, since the effectiveness of a transverse field-based depolarizer depends on the energy and azimuth of its location through the spin response function $F^{\nu}$.

Observation of polarization in the described system is possible in two ways. The first is the resonant depolarization. When the depolarizer frequency is scanned, at the moment of polarization breaking, a jump occurs in the rate of counting the Touschek electrons. The magnitude of the depolarization jump is determined by the square of the polarization degree. To eliminate the influence of the instability of the beam parameters, we detect the ratio of the counting rates from the polarized bunch and unpolarized one. Depending on the scanning parameters, the accuracy of determination of the particle energy from the depolarizer frequency, associated with the jump, reaches $10^{-6}$. The VEPP-4M-KEDR collaboration has a lot of experience in using this method to measure the masses of various particles [17]. In particular, the masses of $J / \psi$ and $\psi^{\prime}$ were measured with an accuracy better than the world average.

Another way is to observe the process of relaxation of the rate of counting particles under conditions when a relatively slow depolarization of the beam occurs because of internal factors rather than because of a resonant external influence. In our case, such a factor is quantum fluctuations in the presence of noticeable stationary perturbations of the guide field. Below we describe this method in more detail.

The relaxation time of polarization ("polarization lifetime") is measured from the time evolution of the Touschek particle counting rate. Correct determination of the polarization lifetime takes into account the Touschek beam lifetime, the spin dependence of the IBS, and scattering on the residual gas by solving the equation for the beam particle population $N(t)$ :

$$
-\frac{d N}{d t}=\frac{1}{\tau_{\mathrm{tsh}}} \frac{N^{2}(t)}{N(0)} \frac{V(0)}{V(t)}[1-\delta(t)]+\frac{N(t)}{\tau_{\mathrm{bg}}} .
$$

Here, the first term corresponds to the IBS and $\tau_{\text {tsh }}$ is the characteristic Touschek beam lifetime; the second term with $\tau_{\text {bg }}$, the characteristic background lifetime, describes background scattering on the residual gas; $\delta(t)$ is the polarization contribution to IBS, proportional to the square of the degree of polarization; $V(t)$ is the beam volume.

During the experiment, the beam volume $V$ (more precisely, the transverse beam sizes because it is assumed that the longitudinal size varies slightly), the beam currents $I_{\mathrm{pol}}, I_{\text {unpol }}$ and the counting rates $f_{\mathrm{pol}}$, $f_{\text {unpol }}$ for the first (polarized) and the second (unpolarized) bunches are measured. The relative count rate difference $\delta f(t)=f_{\text {pol }} / f_{\text {unpol }}-1$ is calculated. These experimental 
data are fitted using the following formulas, which are a solution to (7):

$$
\begin{aligned}
& f_{i}(t)=\frac{p_{\mathrm{tsh}}}{\tau_{\mathrm{tsh}}} \frac{I_{i}^{2}(t)}{I_{i}(0) e f_{0}} \frac{1+\alpha_{V} I_{i}(0)}{1+\alpha_{V} I_{i}(t)}\left[1-\delta_{i}(t)\right]+\frac{p_{\mathrm{bg}}}{\tau_{\mathrm{bg}}} \frac{I_{i}(t)}{e f_{0}} \\
& I_{i}(t)=\frac{I_{i}(0) e^{-t / \tau_{\mathrm{bg}}}}{1+\left(1-e^{-t / \tau_{\mathrm{bg}}}\right) \frac{\tau_{\mathrm{bg}}}{\tau_{\mathrm{shh}}}-\int_{0}^{t} e^{-t / \tau_{\mathrm{bg}}}\left[\delta_{i}(t)+\delta V_{i}(t)\right] \frac{d t}{\tau_{\mathrm{shh}}}} \\
& V(t)=V_{0}\left(1+\frac{\alpha_{V}}{2}\left[I_{1}(t)+I_{2}(t)\right]\right) \\
& \delta f(t)=\frac{f_{\text {pol }}}{f_{\text {unpol }}}-1 \approx-\epsilon(t, 1) e^{-t / \tau_{\mathrm{bg}}}\left[\delta_{\mathrm{pol}}(t)-\delta_{\text {unpol }}(t)\right] \\
& +\frac{\epsilon(t, 1)}{\epsilon(t, 2)} \frac{\delta N+\int_{0}^{t} e^{-t / \tau_{\mathrm{bg}}}\left[\delta_{\mathrm{pol}}(t)-\delta_{\mathrm{unpol}}(t)\right] d t / \tau_{\mathrm{tsh}}}{1+\left(1-e^{-t / \tau_{\mathrm{bg}}}\right) \tau_{\mathrm{bg}} / \tau_{\mathrm{tsh}}} .
\end{aligned}
$$

Here, the index $i$ marks the polarized and unpolarized bunches; $\epsilon(t, j)$ is the factor taking into account the relative probabilities $p_{\text {tsh }}$ and $p_{\text {bg }}$ of registration of the Touschek particles and the beam particles scattered by the residual gas, respectively:

$$
\epsilon(t, j)=\left[\frac{p_{\mathrm{bg}}}{p_{\mathrm{tsh}}}\left(1+\frac{\tau_{\mathrm{tsh}}}{\tau_{\mathrm{bg}}}\right)+\left(j-\frac{p_{\mathrm{bg}}}{p_{\mathrm{tsh}}}\right) e^{-t / \tau_{\mathrm{bg}}}\right]^{-1} ;
$$

$e f_{0}$ is the current of a single electron; $\delta N=I_{\mathrm{pol}}(0) /$ $I_{\text {unpol }}(0)-1$ is the relative difference in the particle population of the bunches. We introduce a time-dependent correction $\delta V_{i}(t)$ to the volume of bunches, using its dependence on the current in the linear approximation:

$$
\begin{aligned}
\delta V_{i}(t) & =\alpha_{V}\left[I_{i}(t)-I_{i}(0)\right] \\
& \approx-\alpha_{V} I_{i}(0) \frac{\left(1-e^{-t / \tau_{\mathrm{bg}}}\right)\left(\tau_{\mathrm{tsh}}+\tau_{\mathrm{bg}}\right)}{\tau_{\mathrm{tsh}}+\tau_{\mathrm{bg}}\left(1-e^{-t / \tau_{\mathrm{bg}}}\right)} .
\end{aligned}
$$

After the end of the acceleration process, the state of polarization of both bunches changes with time. In this connection, their polarization contributions to the counting rate of the Touschek particles are described by the equations

$$
\begin{aligned}
\delta_{\mathrm{pol}}(t) & =\eta\left[P e^{-t / \tau_{d}}+P_{\infty}\left(1-e^{-t / \tau_{d}}\right)\right]^{2} \\
\delta_{\text {unpol }}(t) & =\eta\left[P_{\infty}\left(1-e^{-t / \tau_{d}}\right)\right]^{2}
\end{aligned}
$$

Here, $\eta$ is the Touschek polarization factor; $\tau_{d}$ is the radiative depolarization time; $P$ is the residual degree of polarization of the polarized bunch at the end of acceleration, and $P_{\infty}=(8 \sqrt{3} / 15) \tau_{d} / \tau_{p} \approx 4 \times 10^{-3}$ is the equilibrium polarization degree at $t \rightarrow \infty$.

The following free parameters are used for the fitting: $\Delta=\eta P^{2} \approx 1 \%$ is the polarization Touschek effect; $\tau_{d}$ is the polarization lifetime, which is an object of interest; $\tau_{\text {tsh }} \approx 8000 \mathrm{~s}$ is the Touschek lifetime; $\tau_{\text {bg }} \approx 30000 \mathrm{~s}$ is the background lifetime; $p_{\text {tsh }} \approx 0.2$ is the relative probability of detection of Touschek particles; $p_{\text {bg }} \approx 0.05$ is the relative probability of detection of particles scattered by the residual gas; $I_{\text {pol }}(0) \approx I_{\text {unpol }}(0) \approx 2 \mathrm{~mA}$ are the initial currents of the first and second bunches, respectively; $V_{0}$ is the initial beam volume; $\alpha_{V} \approx 0.1 \% / \mathrm{mA}$ is the coefficient of dependence of the beam volume on the beam current.

Time evolution of the measured quantity $\delta f(t)$ in the conditions when the acceleration to the target energy was just done, but the compensating solenoids stay switched off can be described as follows. At the advance energy, the ratio of the bunch currents is adjusted to a level of $\delta N \approx$ $1 \div 2 \%$ because of the necessity to minimize the slope of the dependence $\delta f(t)$ as a whole and the associated systematic error. For this purpose, we kick out portion by portion the redundant bunch particles using the VEPP$4 \mathrm{M}$ inflector. If the bunch current ratio mentioned above is provided, then $\delta f(t)>0$ during all of the time of observation. The depolarization process is enhanced during crossing of the critical energy area and goes on after completion of the acceleration to the target energy. For this reason, the quantity $\delta f(t)$ grows somewhat exponentially in the positive direction. The characteristic time of this growth for a given target energy is calculated (see Fig. 5). The polarization in the beam drops to zero and then another process becomes dominating - relaxation due to the difference in the bunch currents. Because of the difference in the IBS beam lifetime, the quantity $\delta f(t)$ begins to change in the negative direction. Asymptotically, it goes to zero.

\section{EXPERIMENTAL RESULTS}

One of the RD beam energy calibrations in the advance energy mode is presented in Fig. 8(a). Based on these data, one can get an idea of the magnitude of the polarization effect measured with the Touschek polarimeter. The time allotted for the radiative polarization at the VEPP-3 booster ring at the energy $E=1.65 \mathrm{GeV}$ was $5000-6000 \mathrm{~s}$ at the estimated characteristic time of polarization $\tau_{p} \approx 4000 \mathrm{~s}$. On average, the depolarization jump on the advance energy was $\Delta_{0}=0.99 \pm 0.15 \%$.

First of all, the efficiency of the method was verified by observing the process of beam polarization relaxation (depolarization) after the acceleration at the point $E=$ $1.81 \mathrm{GeV}$ was completed, but the antisolenoids remained switched off. The observed relaxation may indicate that the polarization in the beam is conserved by the end of the acceleration. The time evolution of the normalized Touschek electron counting rate is shown in Fig. 9. In accordance with (7), the fit of the experimental points takes into account the contributions of two processes. One is the radiative depolarization with the characteristic time $\tau_{d}$. The other is the relaxation because of the Touschek losses 


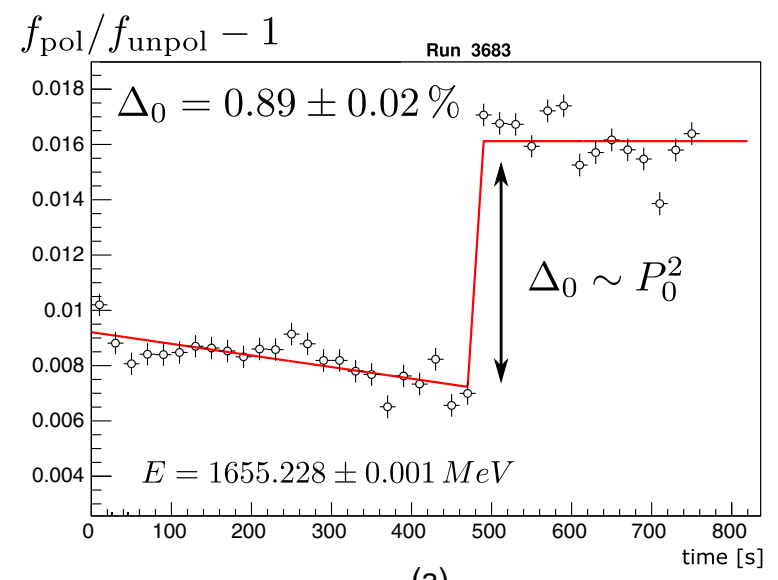

(a)

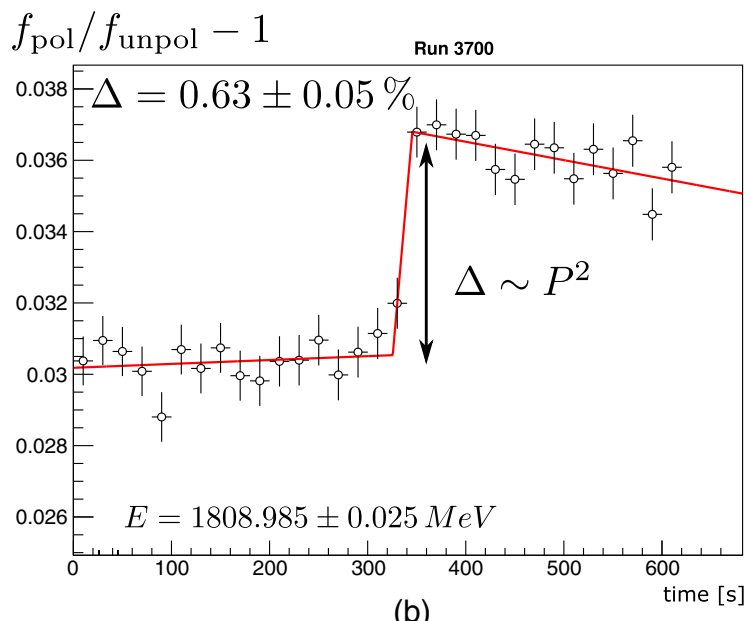

(b)

FIG. 8. Depolarization jumps in two typical scans of the depolarizer frequency: at the advance energy $1655 \mathrm{MeV}$ (a), i.e., before acceleration, and at the target energy $1809 \mathrm{MeV}$ after acceleration with a rate of $2.4 \mathrm{MeV} / \mathrm{s}$ (b). In the second case, before scanning, the compensatory solenoid field was restored in $385 \mathrm{~s}$. Each graph additionally shows the values of the measured depolarization jump and the beam energy, as well as the corresponding errors.

of particles provided that the polarized and unpolarized bunches are not equal in population. In the limit, the observed characteristic $f_{\text {pol }} / f_{\text {unpol }}-1$ tends to zero due to the natural leveling of the bunches. Qualitatively, the relaxation process proceeds as described in the previous section. The relaxation (depolarization) time $\tau_{d}=1470 \pm$ $120 \mathrm{~s}$ determined from the data in Fig. 9 is in good agreement with the estimated time of about $1400 \mathrm{~s}$ (see Fig. 5).

The fact of beam polarization preservation has been fully confirmed in the runs on the beam energy measurement by the resonant depolarization technique applied after the acceleration [Fig. 8(b)].

In contrast to the "spin relaxation" runs, the storage ring mode with the antisolenoid field switched on was restored before every start of the RD procedure. This measure stops the nonresonant (radiative) depolarization process related

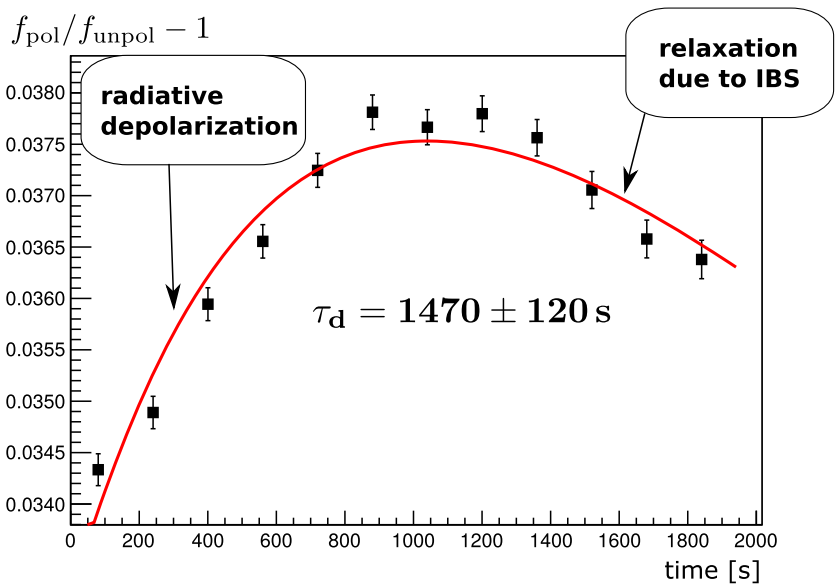

FIG. 9. The process of beam polarization relaxation following the acceleration from the advance energy up to $1806 \mathrm{MeV}$ with a rate of $5 \mathrm{MeV} / \mathrm{s}$. The compensatory solenoids remain switched off. In the time diagram of the ratio of the IBS rates in the polarized bunch and unpolarized one, differing in the number of particles, two stages are clearly distinguished.

to the contribution of strong longitudinal magnetic fields to the spin-orbit coupling. An additional time of $385 \mathrm{~s}$ was required to restore the antisolenoid field and, concurrently, to make necessary corrections to the collider magnetic structure. In accordance with the calculation, if the acceleration stops at $1810 \mathrm{MeV}$ with $P / P_{0}=0.84$, then the relative degree falls down to 0.77 in $385 \mathrm{~s}$. In general, the estimated degradation of the depolarization jump $\Delta \sim P^{2}$ is $\left(P / P_{0}\right)^{2} \approx 0.6$ in magnitude.

At the same time, the square root of the ratio of depolarization jumps measured in several runs before and after acceleration is $\sqrt{\Delta / \Delta_{0}} \approx 0.76 \pm 0.12$. Taking into account the above additional degradation of polarization during the antisolenoid field recovery time, the result of the experiment to intersect an imperfection spin resonance at a rate of $2.4 \mathrm{MeV} / \mathrm{s}$ is characterized by the degree of polarization conservation $P / P_{0}=0.83 \pm 0.13$. Comparing all data in Fig. 6, one can conclude that the experiment and the calculation are in satisfactory quantitative agreement.

\section{DISCUSSION}

The partial Siberian snake technique was first tested with protons at Indiana University Cyclotron Facility [30] and is currently used in routine operation of Brookhaven National Laboratory's Alternating Gradient Synchrotron [31]. The specificity of its application to electron-positron storage rings is the additional complication associated with the depolarizing effect of synchrotron radiation.

Prior to our experiment at VEPP-4M, only one similar experiment was conducted, at VEPP-2M [32]. The radiative polarization was carried out at $600 \mathrm{MeV}$. Then the beam energy was lowered to $380 \mathrm{MeV}$. The polarization 
was preserved due to the adiabatic crossing of the integer spin resonance at $440 \mathrm{MeV}(\nu=1)$ at a rate of $10 \mathrm{MeV} / \mathrm{s}$ using a solenoid described in [14]. In our opinion, [32] and our work differ substantially in analysis of the experimental conditions. In [32], the authors considered the adiabatic condition as decisive. It was briefly noted that the criterion for high-rate crossing relative to the rate of radiative depolarization was satisfied. At the same time, no attention was paid to the fact of the significant spin tune shift. For the parameters of the solenoid at VEPP-2M, the angle of spin rotation in it could be 0.2 radians. This yields a shift from the integer resonance of about $12 \mathrm{MeV}$. Neither the rigorous estimate of the radiative depolarization rate taking into account changes of an equilibrium polarization direction, nor its comparison with the experiment were made.

An interesting experiment [33] on the acceleration of polarized electrons from 1.2 to $3.5 \mathrm{GeV}$ without using the partial Siberian snake method was performed at the Bonn Electron Stretcher Accelerator (ELSA). The polarization was measured in the extracted beams using the Moeller polarimeter, which is sensitive to the sign of polarization. The ramping rate was varied between 0.1 and $7 \mathrm{GeV} / \mathrm{s}$, which is orders of magnitude greater than the rate possible with the nonlaminated magnets at VEPP-4M. The polarization was preserved at acceleration up to $1.9 \mathrm{GeV}$ in the spin-flip (adiabatic) mode with respect to the imperfection resonance crossing. According to the data presented, in ELSA this should take place in the working range of the ramping rate from 0.1 to $5 \mathrm{GeV} / \mathrm{s}$. In contrast to our case, due to the much higher ramping rate, the difficulties in maintaining the polarization in the adiabatic mode, caused by the influence of radiation and noted in Sec. II, could be avoided.

The complete Siberian snake technique $(\varphi=\pi)$ requires a much greater integral of magnetic field: $4.6 \nu$ Tesla $\times$ meter. The corresponding spin tune shift is $1 / 2$, or about $220 \mathrm{MeV}$. Thus, detuning from the integer spin resonances is maximum at any energy. The radiative depolarization factor is approximately $\tau_{p} / \tau_{d} \approx(11 / 54) \pi^{2} \nu^{2}$. With approaching integer spin resonances, the partial Siberian snake solenoid $(\varphi \ll 1)$ yields stronger spin-orbit coupling than the Siberian snake solenoid does. In accordance with (5) and (6), the ratio of the respective depolarization times is $(\nu=k)$

$$
\frac{\tau_{d}(\varphi=\pi)}{\tau_{d}(\varphi \ll 1)} \approx \frac{12}{\varphi^{2}} .
$$

In the VEPP-4M experiment, the longitudinal field integral is $0.6 \times 3.3 \approx 2$ Tesla $\times$ meter against 18.4 Tesla $\times$ meter in the Siberian snake option. The ratio of the depolarization times is about 102: $\tau_{d}(\varphi=\pi)=2.7 \mathrm{~h}$ and $\tau_{d}(\varphi=0.34 \mathrm{rad})=$ $97 \mathrm{~s}$. With the distance from the integer resonance, the ratio of the relaxation times for the cases of strong and weak solenoids is reversed in a qualitative sense. To see this, compare, for example, the curves in Fig. 5 for the 50\% and
$100 \%$ decompensation of the detector field. As applied to our case, a complete Siberian snake could be better since it eliminates all possible spin resonances and has a much smaller depolarizing effect at the resonance energy. At the same time, economically, the method based on decompensation of the longitudinal field of the detector cost us nothing. With its help we easily solved the practical problem that arose during our experiment on high energy physics.

At VEPP-4M, the radiative depolarization can limit the efficiency of the method developed with growth of the target beam energy. Estimates show that it is possible to intersect the third resonance $E=1322 \mathrm{MeV}$ practically without polarization loss during beam deceleration with a rate of $2 \mathrm{MeV} / \mathrm{s}$ starting from $1550 \mathrm{MeV}$. However, acceleration in the same manner from $1.85 \mathrm{GeV}$ up to $2.4 \mathrm{GeV}$, while crossing the fifth resonance $E=2203 \mathrm{MeV}$, will lead to a threefold decrease in the polarization degree. In this case, we plan to increase the ramping rate to $10-20 \mathrm{MeV}$ per second. In addition, it will be necessary to study the possibilities of crossing the resonances $3-\nu_{x, z}, 4+\nu_{x, z}$ and $5-\nu_{x, z}$ falling within the specified ranges.

[1] V. Anashin et al., The KEDR detector, Phys. Part. Nucl. 44, 657 (2013).

[2] V. Anachin et al., in Proceedings of the 6th European Particle Accelerator Conference, Stockholm, 1998 (IOP, London, 1998), pp. 400-402.

[3] M. M. V. Dyug, A. V. Grigoriev, V. A. Kiselev, B. A. Lazarenko, E. B. Levichev, A. I. Mikaiylov, S. I. Mishnev, S. A. Nikitin, D. M. Nikolenko, I. A. Rachek, Yu. V. Shestakov, D. K. Toporkov, S. A. Zevakov, and V. N. Zhilich, Møller polarimeter for VEPP-3 storage ring based on internal polarized gas jet target, Nucl. Instrum. Methods Phys. Res., Sect. A 536, 338 (2005).

[4] V. V. Anashin, V. Aulchenko, I. Nikolaev et al., Measurement of the $\tau$ lepton mass at the KEDR detector, JETP Lett. 85, 347 (2007).

[5] M. Froissart and R. Stora, Depolarisation d'un faisceau de protons polarises dans un synchrotron, Nucl. Instrum. Methods 7, 297 (1960).

[6] Ya.S. Derbenev and A. M. Kondratenko, Relaxation and equilibrium state of electron polarization in accumulators, Dokl. Akad. Nauk SSSR 217, 311 (1974).

[7] A. Bogomyagkov, S. Nikitin, I. Nikolaev, V. Kiselev, E. Kremyanskaya, E. Levichev, E. Simonov, and A. Skrinsky, in Proceedings of the 9th European Particle Accelerator Conference, Lucerne, 2004 (EPS-AG, Lucerne, 2004).

[8] Ya. S. Derbenev, A. M. Kondratenko, and A. N. Skrinsky, Radiative polarization at ultra-high energies, Part. Accel. 9, 247 (1979).

[9] Ya. S. Derbenev and A. M. Kondratenko, Polarization kinetics of particles in storage rings, Sov. Phys. JETP 37, 968 (1973).

[10] A. A. Sokolov and I. M. Ternov, Polarization and spin effects in the synchrotron radiation theory, Dokl. Akad. Nauk SSSR 153, 1052 (1963). 
[11] A. M. Kondratenko, Polarized beams in storage rings and cyclic accelerators, Doctoral thesis, Budker Institute of Nuclear Physics, Novosibirsk, USSR, 1982.

[12] S. Nikitin, Report No. 2015-1, Budker Institute of Nuclear Physics, Novosibirsk, 2015.

[13] A. Barladyan et al., in Proceedings of the International Particle Accelerator Conference (IPAC'17), Copenhagen, Denmark, 2017, International Particle Accelerator Conference No. 8 (JACoW, Geneva, Switzerland, 2017), pp. 4451-4453, https://doi.org/10.18429/JACoWIPAC2017-THPVA017.

[14] Ya. S. Derbenev, A. M. Kondratenko, S. I. Serednyakov, A. N. Skrinsky, G. M. Tumaikin, and Yu. M. Shatunov, Polarized particles in storage rings, in Proceedings, Xth International Conference On High Energy Accelerators (1977), pp. 55-63.

[15] Ya. S. Derbenev, A. M. Kondratenko, S. I. Serednyakov, A. N. Skrinsky, G. M. Tumaikin and Yu. M. Shatunov, Radiative polarization: obtaining, control, using, Part. Accel. 8, 115 (1978).

[16] S. Nikitin and E. Saldin, Report IYaF SO AN SSSR 198119 (1981), Internal Report DESY L-Trans- 290, Hamburg, 1984.

[17] V. E. Blinov et al., High precision energy calibration with resonant depolarization at the VEPP-4M collider, Nucl. Part. Phys. Proc. 273-275, 210 (2016).

[18] S. Nikitin, A concept of obtaining longitudinal polarization at the JINR tau-charm factory, Nucl. Instrum. Methods Phys. Res., Sect. A 378, 495 (1996).

[19] S. Nikitin, in the 20th Russian Conference on Charged Particle Accelerators (RuPAC 2006) (Novosibirsk, Russian Federation, 2006), p. MOAP01.

[20] S. Nikitin, Radiative relaxation time of longitudinal polarization in the $2 \times 3 \times 5.7^{0}$ central arc scheme for SuperB, arXiv:1909.04310.

[21] K. Steffen, Internal DESY Report No. HERA82/11, 1982.

[22] S. Nikitin and I. Protopopov, Report No. BINP SB RAS 1999-44, 1999.

[23] S. Nikitin and E. Simonov, Beam-beam effects with strong betatron coupling at interaction point, in 2nd Asian Particle
Accelerator Conference, Beijing, China, 2001, pp. WEP061, https://cds.cern.ch/record/914729?ln=ru.

[24] V. Blinov, A. Bogomyagkov, I. Nikolaev, S. Karnaev, V. Kiselev, B. Levichev, E. Levichev, O. Meshkov, S. Mishnev, A. Naumenkov, S. Nikitin, V. Popov, A. Polunin, I. Protopopov, D. Shatilov, E. Simonov, Y. Tikhonov, and G. Tumaikin, Absolute calibration of particle energy at VEPP-4M, Nucl. Instrum. Methods Phys. Res., Sect. A 494, 81 (2002).

[25] V. E. Blinov, A. V. Bogomyagkov, I. B. Nikolaev, N. Y. Muchnoi, S. A. Nikitin, A. G. Shamov, and V. N. Zhilich, Review of beam energy measurements at VEPP-4M collider: KEDR/VEPP-4M, Nucl. Instrum. Methods Phys. Res., Sect. A 598, 23 (2009).

[26] V. N. Bayer and V. A. Khoze, Determination of the transverse polarization of high-energy electrons, Yad. Fiz. 9, 409 (1969) [Sov. J. Nucl. Phys. 9, 238 (1969)].

[27] V. N. Bayer, V. M. Katkov, and V. M. Strakhovenko, On the influence of polarization upon the effects of internal electron scattering in accumulators, Dokl. Akad. Nauk SSSR 241, 797 (1978).

[28] V. M. Strakhovenko, Coulomb effects in the spindependent contribution to the intrabeam scattering rate, Phys. Rev. ST Accel. Beams 14, 012803 (2011).

[29] S. A. Nikitin and I. B. Nikolaev, Calculation of the intensity of Touschek electrons in the VEPP-4M storage ring, J. Exp. Theor. Phys. 115, 36 (2012).

[30] T. Roser, First experimental test of the siberian snake concept, in High Energy Spin Physics, edited by K.-H. Althoff and W. Meyer (Springer-Verlag, Berlin, 1991), pp. 284-291.

[31] H. Huang et al., Preservation of Proton Polarization by a Partial Siberian Snake, Phys. Rev. Lett. 73, 2982 (1994).

[32] L. Barkov et al., Measurement of the properties of the $\Omega$ meson with a cryogenic magnetic detector, JETP Lett. 46, 164 (1987).

[33] S. Nakamura et al., Acceleration of polarized electrons in ELSA, Nucl. Instrum. Methods Phys. Res., Sect. A 411, 93 (1998). 